\title{
Extensive next-generation sequencing analysis in chronic lymphocytic leukemia at diagnosis: clinical and biological correlations
}

Gian Matteo Rigolin ${ }^{1 *+}$ (D) Elena Saccenti ${ }^{1+}$, Cristian Bassi $^{2}$, Laura Lupini ${ }^{2}$, Francesca Maria Quaglia ${ }^{1}$, Maurizio Cavallari ${ }^{1}$, Sara Martinelli', Luca Formigaro ${ }^{1}$, Enrico Lista', Maria Antonella Bardi ${ }^{1}$, Eleonora Volta', Elisa Tammiso ${ }^{1}$, Aurora Melandri ${ }^{1}$, Antonio Urso ${ }^{1}$, Francesco Cavazzini ${ }^{1}$, Massimo Negrini ${ }^{2+}$ and Antonio Cuneo ${ }^{1+}$

\begin{abstract}
Background: In chronic lymphocytic leukemia (CLL), next-generation sequencing (NGS) analysis represents a sensitive, reproducible, and resource-efficient technique for routine screening of gene mutations.

Methods: We performed an extensive biologic characterization of newly diagnosed CLL, including NGS analysis of 20 genes frequently mutated in CLL and karyotype analysis to assess whether NGS and karyotype results could be of clinical relevance in the refinement of prognosis and assessment of risk of progression. The genomic DNA from peripheral blood samples of 200 consecutive CLL patients was analyzed using lon Torrent Personal Genome Machine, a NGS platform that uses semiconductor sequencing technology. Karyotype analysis was performed using efficient mitogens.

Results: Mutations were detected in $42.0 \%$ of cases with $42.8 \%$ of mutated patients presenting 2 or more mutations. The presence of mutations by NGS was associated with unmutated IGHV gene $(p=0.009)$, CD38 positivity $(p=0.010)$, risk stratification by fluorescence in situ hybridization (FISH) $(p<0.001)$, and the complex karyotype $(p=0.003)$. A high risk as assessed by FISH analysis was associated with mutations affecting TP53 ( $p=0.012), B I R C 3(p=0.003)$, and FBXW7 $(p=0.003)$ while the complex karyotype was significantly associated with TP53, ATM, and MYD88 mutations ( $p=0.003$, 0.018 , and 0.001 , respectively). By multivariate analysis, the multi-hit profile ( $\geq 2$ mutations by NGS) was independently associated with a shorter time to first treatment $(p=0.004)$ along with TP53 disruption $(p=0.040)$, IGHV unmutated status $(p<0.001)$, and advanced stage $(p<0.001)$. Advanced stage $(p=0.010)$, TP53 disruption $(p<0.001)$, IGHV unmutated status $(p=0.020)$, and the complex karyotype $(p=0.007)$ were independently associated with a shorter overall survival.
\end{abstract}

Conclusions: At diagnosis, an extensive biologic characterization including NGS and karyotype analyses using novel mitogens may offer new perspectives for a better refinement of risk stratification that could be of help in the clinical management of CLL patients.

Keywords: Chronic lymphocytic leukemia, Gene mutation analysis, Next-generation sequencing, Complex karyotype, Prognosis

\footnotetext{
* Correspondence: rglgmt@unife.it

'Equal contributors

${ }^{1}$ Hematology Section, Department of Medical Sciences, Azienda

Ospedaliero-Universitaria, Arcispedale S. Anna, University of Ferrara, Via Aldo

Moro, 8, 44124 Ferrara, Cona, Italy

Full list of author information is available at the end of the article
} 


\section{Background}

Chronic lymphocytic leukemia (CLL) displays a heterogeneous clinical course [1-3], some patients living for years with asymptomatic disease and others experiencing early progression requiring therapeutic intervention. Modern treatment algorithms must take into account age, comorbidities, and prognostic/predictive factors, including genetic lesions [4]. Adverse prognostic factors include stage [5], positivity for CD38, ZAP70, and CD49d [6-8], and, among genetic features, the unmutated configuration of the variable region of the immunoglobulin heavy chain gene $(I G H V)[6]$ and specific molecular cytogenetic lesions revealed by fluorescent in situ hybridization (FISH). More recently, karyotype aberrations were shown to represent strong prognostic factors [9-14], and large retrospective studies demonstrated that TP53, NOTCH1, and SF3B1 gene mutations have a negative impact on the time to first treatment (TTFT) and overall survival (OS) [15-17]. These data were in part confirmed by prospective clinical trials using homogeneous treatment protocols [18, 19], and recurrent genomic lesions were included within comprehensive prognostic indexes [20,21] helping clinicians to counsel patients more appropriately, to define the follow-up interval, and, potentially, to provide a rational basis to design early intervention protocols for high-risk patients [22].

Next-generation sequencing (NGS) techniques documented that, besides the aforementioned genes, a number of previously unidentified genes may be mutated in CLL and that the disruption of putative core cellular pathways represents an important mechanism promoting disease progression and drug resistance [23-26]. NGS may detect minor cell populations (subclones) harboring a variety of gene mutations, including NOTCH1, SF3B1, BIRC3, and TP53 mutations, the latter having a negative prognostic impact that was similar to TP53 clonal mutations [27-29] as detected by conventional sequencing techniques (i.e., Sanger sequencing).

Thus, NGS is becoming of age for usage in clinical practice, and indeed, over $50 \%$ of CLL patients were shown to carry mutations in one or more genes [30,31], potentially making NGS a sensitive tool for the detection of mutations including subclonal mutations.

To assess whether an extended mutational screening by NGS at diagnosis could allow for a refinement of our capability to predict TTFT and OS, we designed a CLLspecific gene panel, covering hotspots or complete coding regions of 20 genes more frequently mutated in CLL. We performed NGS of these 20 genes using a resourceefficient platform in 200 consecutive newly diagnosed patients representing over $90 \%$ of CLL incident cases in our region. By correlating mutational data obtained by an extensive genetic/cytogenetic characterization with clinicbiological parameters and outcome, we were able to show that NGS screening was an independent prognostic factor for TTFT and that complex karyotype was a strong predictor of an inferior survival in this patient population.

\section{Methods \\ Patients}

The study cohort consisted of 200 consecutive untreated CLL patients diagnosed and followed between 2007 and 2014. All patients were diagnosed according to NCI criteria [32]. Only patients with a Matutes immunophenotypic score [33] $\geq 3$ (i.e., typical CLL) were included. CD38 and ZAP-70 were tested on peripheral blood (PB) cells, as described [34]. When needed, mantle cell lymphoma was excluded by the evaluation of cyclin D1. The study was approved by the local ethics committee. Indications for treatment included increased white blood cell count with $<6$ month lymphocyte doubling time, anemia or thrombocytopenia due to bone marrow infiltration or autoimmune phenomena not responding to steroids, and disease progression in the Binet staging system. Fludarabine and bendamustine (since 2010), containing regimens in association with or without rituximab, were used as first-line treatment; chlorambucil was used in elderly and unfit patients according to shared treatment policy adopted at our center.

\section{Cytogenetic and FISH analyses}

Interphase FISH was performed on PB samples obtained at diagnosis using probes for the following regions: 13q14, 12q13, 11q22/ATM, and 17p13/TP53 (Vysis/Abbott Co, Downers Grove, IL) as described [35]. Each patient was categorized into a FISH risk group according to the following classification: favorable group (isolated 13q14 deletion or absence of FISH aberrations), unfavorable group (deletions of 11q22 or of $17 \mathrm{p} 13$ ), and intermediate group (trisomy 12).

Cytogenetic analysis was performed on the same samples used for FISH analysis using CpG-oligonucleotide DSP30 $(2 \mu \mathrm{mol} / \mathrm{l}$ TibMolBiol Berlin, Germany) plus IL2 (100 U/ml Stem Cell Technologies Inc., Milan, Italy) as described [36]. The complex karyotype was defined by the presence of at least 3 chromosome aberrations.

\section{IGHV analysis}

$I G H V$ genes were amplified from genomic DNA and sequenced according to standard methods with the cutoff of $98 \%$ homology to the germline sequence to discriminate between mutated $(<98 \%)$ and unmutated $(\geq 98 \%)$ cases, as reported [35].

\section{Ion Torrent Personal Genome Machine (PGM) analysis}

NGS analysis was performed on the same samples used for FISH and cytogenetic analyses. In all samples, the percentage of CLL cells was over $90 \%$ as assessed by 
flow cytometry analysis. Agilent HaloPlex Target Enrichment kit (Agilent Technologies, Santa Clara, CA, USA) was used to produce libraries of exonic regions from 20 genes (ATM, BIRC3, BRAF, CDKN2A, PTEN, CDH2, DDX3X, FBXW7, KIT, KLHL6, KRAS, MYD88, NOTCH1, NRAS, PIK3CA, POT1, SF3B1, TP53, XPO1, ZMYM3) starting from genomic DNA from $\mathrm{PB}$ samples, according to HaloPlex Target Enrichment System (Agilent Technologies, Santa Clara, CA, USA). Diluted libraries were linked to Ion Sphere Particles, clonally amplified in an emulsion PCR and enriched using Ion OneTouch emulsion PCR System (Life technologies, Foster City, CA, USA). Exon-enriched DNA was precipitated with magnetic beads coated with streptavidin. Enriched, templatepositive Ion Sphere Particles were loaded in one ion chip and sequenced using Ion Torrent PGM (Life technologies, Foster City, CA, USA). Sequencing data were aligned to the human reference genome (GRCh37). Data analysis and variant identification were performed using Torrent Suite 3.4 and Variant Caller plugin 3.4.4 (Life technologies, Foster City, CA, USA) [37].

\section{Statistical analysis}

The Mann-Whitney and the Pearson's chi-squared tests were applied for quantitative and categorical variables, respectively. TTFT was calculated as the interval between diagnosis and the start of first-line treatment. OS was calculated from the date of diagnosis until death due to any cause or until the last patient follow-up. Survival curves were compared by the log-rank test. Proportional hazards regression analysis was used to identify the significant independent prognostic variables on TTFT. The stability of the Cox model was internally validated using bootstrapping procedures [15]. Statistical analysis was performed using Stata 14.0 (Stata Corp, College Station, TX).

\section{Results}

Patients and mutation analyses of the $\mathbf{2 0}$ genes by NGS

The clinical and biologic characteristics of the 200 CLL patients are presented in Table 1.

Parallel sequencing of exonic regions from the 20 genes showed somatic mutations in 84/200 (42.0 \%) cases. One hundred thirty-six mutations were found in these 84 patients; 114 missense mutations, 7 nonsense mutations, 14 frameshit deletions, and 1 frameshit insertion. Mutations were detected with a frequency ranging from 5.0 to $96.7 \%$ of the reads. Sixteen cases (8.0\%) showed mutations in the TP53 gene, $16(8.0 \%)$ in the NOTCH1 gene, 15 (7.5\%) in the SF3B1 gene, 10 (5.0\%) in the ATM gene, 8 (4.0\%) in the BIRC3 gene, 7 (3.5\%) in the MYD88 gene, 7 (3.5\%) in the PTEN gene, 6 (3.0\%) in the FBXW7 gene, 5 (2.5\%) in the POT1 gene, 5 (2.5\%) in the BRAF gene, 5 (2.5\%) in the ZMYM3
Table 1 Clinical and biological characteristics of the $200 \mathrm{CLL}$ patients

\begin{tabular}{ll}
\hline Variable & \\
\hline Age, median yrs (range) & $67.6(38.3-89.9)$ \\
Sex m/f & $121 / 79$ \\
Binet stage a/b/c & $161 / 25 / 14$ \\
CD38 neg/pos & $121 / 79$ \\
ZAP-70 neg/pos & $143 / 37$ \\
IGVH mut/unmut & $105 / 91$ \\
13q14 deletion yes/no & $104 / 96$ \\
Trisomy 12 yes/no & $32 / 168$ \\
11 q22 deletion yes/no & $20 / 180$ \\
17p13 deletion yes/no & $9 / 191$ \\
FISH fav/int/unfav & $142 / 30 / 28$ \\
Complex karyotype no/yes & $167 / 28$ \\
Mutated patients by NGS no/yes & $116 / 84$ \\
No. of mutations by NGS 0/1/2/3/4 & $116 / 48 / 24 / 8 / 4$ \\
TP53 mut/WT & $16 / 184$ \\
TP53 disruption yes/no & $19 / 181$ \\
\hline
\end{tabular}

$f$ female, fav favorable, int intermediate, $m$ male, mut mutated, neg negative, pos positive, unfav unfavorable, unmut unmutated, yrs years, TP53 disruption 17p13 deletion and/or TP53 mutation

gene, and 19 (9.5\%) cases in the remaining 9 genes (Additional file 1: Table S1). 36/84 (42.8 \%) mutated patients presented 2 or more mutations (Additional file 2: Table S2). TP53 mutations $(p=0.027)$ were significantly more frequent among patients with 2 or more mutations while a trend was observed for BIRC3 mutations $(p=0.059)$ and mutations of genes less frequently mutated in CLL $(p=0.057)$ (Additional file 3: Table S3).

\section{Correlations between mutational status by NGS, molecular cytogenetic findings, and clinico-biological parameters}

The presence of somatic mutations did not correlate with sex, age, and Binet stage while the occurrence of mutations by NGS analysis was significantly associated with CD38 positivity $(p=0.010), I G H V$ unmutated status $(p=0.009)$, intermediate high-risk cytogenetics by FISH analysis $(p<0.001)$, and the complex karyotype $(p=0.003$; Table 2).

A higher risk as assessed by FISH analysis was associated with the presence of mutations affecting TP53 $(p=0.012)$, BIRC3 $(p=0.003)$, and FBXW7 $(p=0.003)$ while the complex karyotype was significantly associated with TP53, ATM, and MYD88 mutations ( $p=0.003,0.018$, and 0.001 , respectively: Table 3 ; Fig. 1 ).

The median follow-up for the 200 CLL patients was 52.3 months. In univariate analysis (Table 4 ), the occurrence of mutations and the presence of 2 or more 
Table 2 Correlations between mutational status by NGS analysis and clinical biological parameters

\begin{tabular}{llll}
\hline & $\begin{array}{l}\text { Mutated } \\
(n=84)\end{array}$ & $\begin{array}{l}\text { Not mutated } \\
(n=116)\end{array}$ & $p$ \\
\hline Sex m/f & $49 / 35$ & $72 / 44$ & 0.594 \\
Age $<70 / \geq 70$ years & $46 / 38$ & $69 / 47$ & 0.505 \\
Binet stage a/b/c & $66 / 12 / 6$ & $95 / 13 / 8$ & 0.802 \\
CD38 neg/pos & $42 / 42$ & $79 / 37$ & 0.010 \\
IGHV mut/unmut & $36 / 48$ & $69 / 43$ & 0.009 \\
FISH fav/int unfav & $48 / 36$ & $94 / 22$ & $<0.001$ \\
Complex karyotype no/yes & $63 / 19$ & $104 / 9$ & 0.003 \\
\hline
\end{tabular}

$f$ female, fav favorable, int intermediate, $m$ male, mut mutated, neg negative, pos positive, unfav unfavorable, unmut unmutated, yrs years, TP53 disruption $17 \mathrm{p} 13$ deletion and/or TP53 mutation

mutations were significantly associated with a worse TTFT (Fig. 2) along with advanced Binet stage; CD38 positivity; $I G H V$ unmutated status; intermediate unfavorable FISH results; 11q22 deletion, 17p13 deletion, and/or TP53 mutations (here referred to as TP53 disruption); and complex karyotype. A shorter TTFT was also observed for TP53-, NOTCH1-, ATM-, and BRAFmutated patients. By multivariate analysis (Table 5 ), we found that the multi-hit profile ( $\geq 2$ mutations by NGS) predicted a shorter TTFT $(p=0.004)$ along with TP53 disruption $(p=0.040), I G H V$ unmutated status $(p<$ $0.001)$, and advanced stage $(p<0.001)$.

When considering OS (Table 4), a poorer prognosis was associated with the occurrence of mutations by NGS analysis, the presence of 2 or more mutations, with TP53 mutations, and with advanced stage, CD38 positivity, IGHV unmutated status, TP53 disruption, and complex karyotype. In multivariate analysis, advanced stage $(p=0.010), I G H V$ unmutated status $(p=$ $0.020)$, TP53 disruption $(p<0.001)$, and the complex karyotype $(p=0.007)$ independently predicted a worse outcome (Table 5).

\section{Discussion}

CLL is the most frequent leukemia in western countries and has a significant socioeconomic impact. It is therefore important to define which patients are at higher risk of progression and therefore require stricter follow-up and which genetic lesions are associated with risk of relapse and/or chemorefractoriness ultimately determining a shorter survival [22]. Unlike previous reports analyzing prognostic/predictive factors in CLL requiring treatment at the time of progression, we were able to perform an extensive biologic characterization in an unselected prospective series of 200 patients diagnosed over an 8-year span and followed for a median of 52.3 months over the last 10 years. Our center has a >90\% capture of each incident case of CLL in our region of approximately 400,000 inhabitants because the diagnosis of CLL in our province was centralized since 2006 . With the exception of frail patients with a significant number of comorbidities precluding any form of specific treatment, whom were not submitted to extensive molecular cytogenetic characterization, the patient population included in this analysis is highly representative of the true nature of CLL and allows meaningful analyses of TTFT and OS in a real-world scenario.

Table 3 Correlations between mutations by NGS analysis, FISH results, and karyotype complexity

\begin{tabular}{|c|c|c|c|c|c|c|}
\hline & \multicolumn{2}{|c|}{ FISH results } & & \multicolumn{2}{|c|}{ Complex karyotype } & \multirow[b]{2}{*}{$p$} \\
\hline & Fav & Int-unfav & & No & Yes & \\
\hline No. of mutations by NGS no/1/ $\geq 2$ & $94 / 28 / 20$ & $22 / 19 / 17$ & 0.001 & $104 / 36 / 27$ & $9 / 11 / 8$ & 0.011 \\
\hline TP53 WT/mut & $135 / 7$ & $49 / 9$ & 0.012 & $158 / 9$ & $22 / 6$ & 0.003 \\
\hline NOTCH1 WT/mut & $133 / 9$ & $51 / 7$ & 0.175 & $155 / 12$ & $25 / 3$ & 0.517 \\
\hline SF3B1 WT/mut & $132 / 10$ & $53 / 5$ & 0.701 & $156 / 11$ & $25 / 3$ & 0.434 \\
\hline ATM WT/mut & $137 / 5$ & $53 / 5$ & 0.133 & $161 / 6$ & $24 / 4$ & 0.018 \\
\hline BIRC3 WT/mut & $140 / 2$ & $52 / 6$ & 0.003 & $161 / 6$ & $26 / 2$ & 0.381 \\
\hline MYD88 WT/mut & $136 / 6$ & $57 / 1$ & 0.382 & $164 / 3$ & $24 / 4$ & 0.001 \\
\hline PTEN WT/mut & $138 / 4$ & $55 / 3$ & 0.411 & $161 / 6$ & $27 / 1$ & 0.996 \\
\hline FBXW7 WT/mut & $141 / 1$ & $53 / 5$ & 0.003 & $161 / 6$ & $28 / 0$ & 0.308 \\
\hline POT1 WT/mut & $138 / 4$ & $57 / 1$ & 0.653 & $162 / 5$ & $28 / 0$ & 0.354 \\
\hline BRAF WT/mut & $139 / 3$ & $56 / 2$ & 0.583 & $163 / 4$ & $28 / 0$ & 0.408 \\
\hline ZMYM3 WT/mut & $138 / 4$ & $57 / 1$ & 0.653 & $163 / 4$ & $27 / 1$ & 0.716 \\
\hline Others WT/mut & $129 / 13$ & $49 / 6$ & 0.192 & $149 / 18$ & $24 / 4$ & 0.587 \\
\hline
\end{tabular}

$f$ female, fav favorable, int intermediate, $m$ male, mut mutated, neg negative, pos positive, unfav unfavorable, unmut unmutated 


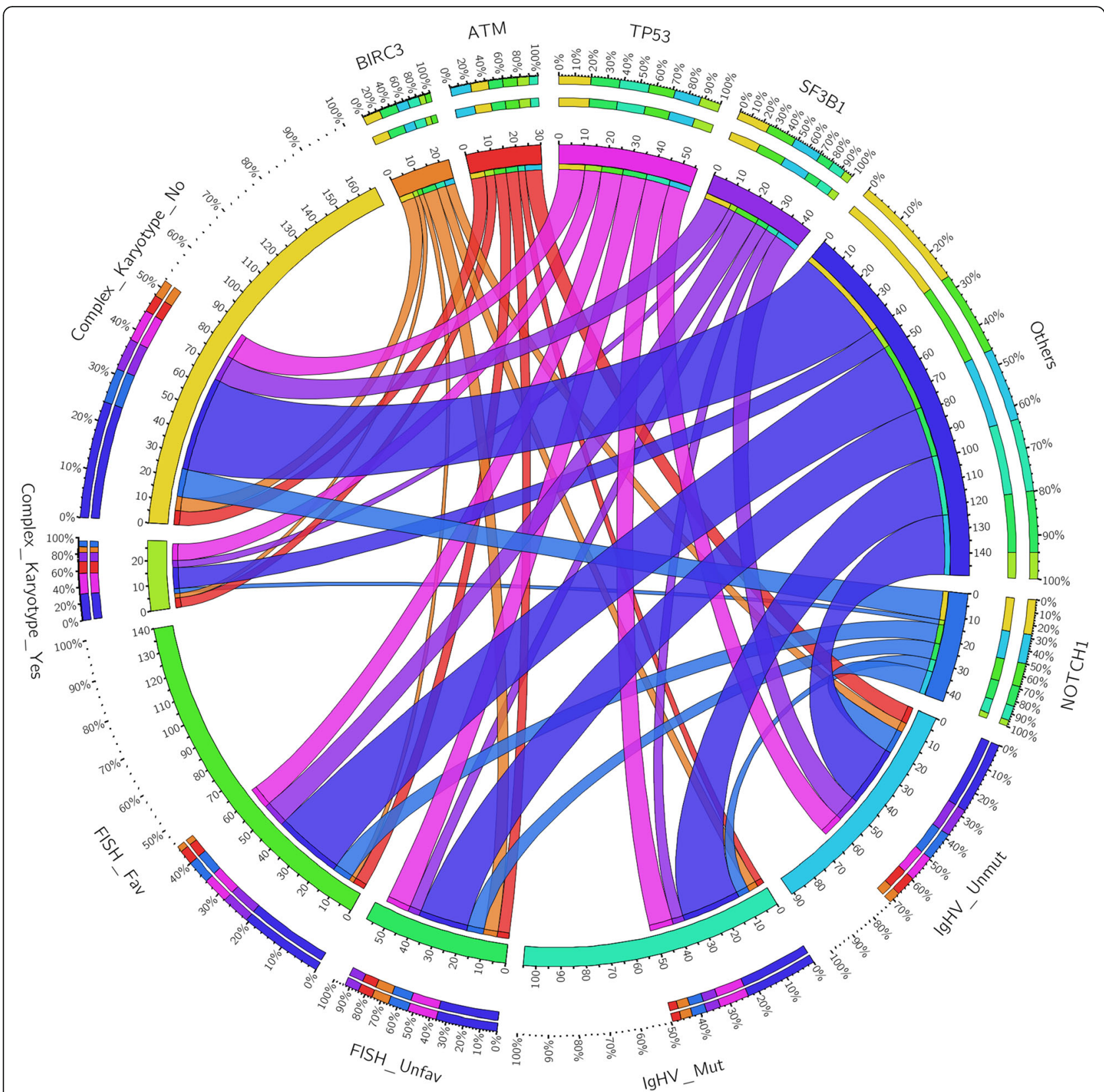

Fig. 1 Gene mutations and correlation with genomic features: circos diagrams illustrating pairwise co-occurrence of gene mutations with IGHV status, FISH results, and complex karyotype

The Ion Torrent PGM is a NGS platform that uses semiconductor sequencing technology. In clinical practice, PGM may represent a very sensitive tool for mutational screening of patients with CLL, allowing multiplexing of samples and gene targets in one experimental setup [30] and resulting in higher speed of analysis and lower costs [38]. Parallel sequencing of exonic regions in these 20 CLL-related genes showed somatic mutations in 84/200 (42.0\%) cases by using a $5 \%$ cutoff. Mutations were detected with a frequency ranging from 5.0 to $96.7 \%$ of the reads, clearly showing that both major and minor clonal mutations were present, the former representing early leukemogenetic events and the latter representing late-appearing aberrations possibly associated with disease progression or chemorefractoriness $[39,40]$.

In this series, the frequency of mutations involving TP53, NOTCH1, SF3B1, ATM, and BIRC3 genes clearly reflects the nature of our patient cohort that included 
Table 4 Univariate analysis for TTFT and OS

\begin{tabular}{|c|c|c|c|c|c|}
\hline \multirow[b]{2}{*}{ Variable } & \multirow[b]{2}{*}{$N$ pts } & \multicolumn{2}{|l|}{ TTFT } & \multicolumn{2}{|l|}{ OS } \\
\hline & & HR (Cl $95 \%)$ & $p$ & HR (CI $95 \%)$ & $p$ \\
\hline Binet stage $B-C$ vs $A$ & 39 vs 161 & $9.884(5.939-16.450)$ & $<0.0001$ & $3.174(1.677-6.007)$ & 0.0002 \\
\hline CD38 pos vs neg & 79 vs 121 & $4.097(2.564-6.546)$ & $<0.0001$ & $3.123(1.686-5.783)$ & 0.0001 \\
\hline IGVH mut vs unmut & 105 vs 91 & $5.584(3.326-9.374)$ & $<0.0001$ & $3.667(1.886-7.127)$ & $<0.0001$ \\
\hline $11 \mathrm{q} 22$ deletion yes vs no & 20 vs 180 & $2.879(1.528-5.426)$ & 0.0006 & $1.736(0.739-4.078)$ & 0.2000 \\
\hline TP53 disruption yes/no & 19 vs 181 & $3.284(1.867-5.781)$ & $<0.0001$ & $4.246(2.076-8.687)$ & $<0.0001$ \\
\hline FISH int-unfav vs fav & 58 vs 142 & $2.605(1.670-4.063)$ & $<0.0001$ & $2.432(1.438-4.454)$ & 0.0029 \\
\hline Complex karyotype yes vs no & 28 vs 167 & $2.979(1.756-5.056)$ & $<0.0001$ & $3.854(1.961-7.578)$ & $<0.0001$ \\
\hline Mutations by NGS no/yes & 116 vs 84 & $2.835(1.799-4.469)$ & $<0.0001$ & $2.171(1.176-4.008)$ & 0.0130 \\
\hline \multicolumn{6}{|l|}{ Number of mutations by NGS } \\
\hline 0 & 116 & 1 & $<0.001$ & 1 & 0.037 \\
\hline 1 & 47 & $2.373(1.369-4.112)$ & $0.002^{\mathrm{a}}$ & $1.936(0.930-4.032)$ & $0.078^{\mathrm{a}}$ \\
\hline$\geq 2$ & 37 & 3.418 (2.009-5.759) & $<0.001^{\mathrm{a}}$ & $2.466(1.187-5.126)$ & $0.016^{\mathrm{a}}$ \\
\hline TP53 mut vs wt & 16 vs 184 & $2.804(1.514-5.194)$ & 0.0010 & $2.793(1.284-6.098)$ & 0.0069 \\
\hline NOTCH1 mut vs wt & 16 vs 184 & $2.353(1.164-4.762)$ & 0.0141 & $2.646(1.114-6.259)$ & 0.0219 \\
\hline SF3B1 mut vs wt & 15 vs 185 & $1.779(0.886-3.571)$ & 0.1006 & $1.170(0.419-3.268)$ & 0.7648 \\
\hline ATM mut vs wt & 10 vs 190 & $3.623(1.715-7.633)$ & 0.0003 & $1.946(0.686-5.525)$ & 0.2023 \\
\hline BIRC3 mut vs wt & 8 vs 192 & $0.817(0.254-2.597)$ & 0.7246 & $1.099(0.252-4.808)$ & 0.8998 \\
\hline MYD88 mut vs WT & 7 vs 193 & $1.758(0.642-4.812)$ & 0.2724 & $1.505(0.363-6.240)$ & 0.5733 \\
\hline PTEN mut vs WT & 7 vs 193 & $1.573(0.574-4.310)$ & 0.3780 & $1.503(0.363-6.224)$ & 0.5742 \\
\hline FBXW7 mut vs WT & 6 vs 194 & $1.820(0.664-4.988)$ & 0.2441 & $1.445(0.349-5.986)$ & 0.6134 \\
\hline POT1 mut vs WT & 5 vs 195 & $1.059(0.259-0.321)$ & 0.9375 & $0.978(0.352-4.768)$ & 0.9973 \\
\hline BRAF mut vs WT & 5 vs 195 & $7.730(3.014-19.827)$ & $<0.0001$ & $2.126(0.286-15.823)$ & 0.4610 \\
\hline ZMYM3 mut vs WT & 5 vs 195 & $0.484(0.067-3.480)$ & 0.4710 & $2.336(0.563-9.693)$ & 0.2434 \\
\hline OTHERS mut vs wt & 19 vs 181 & $1.036(0.517-2.075)$ & 0.9205 & $0.898(0.320-2.518)$ & 0.8381 \\
\hline
\end{tabular}

${ }^{a}$ Compared with no mutation

$f$ female, fav favorable, int intermediate, $m$ male, mut mutated, neg negative, pos positive, unfav unfavorable, unmut unmutated, yrs years, TP53 disruption $17 \mathrm{p} 13$ deletion and/or TP53 mutation

untreated CLL analyzed early during the natural history of the disease and comprising $80.5 \%$ of Binet stage A cases. Approximately, the same incidence for these mutations was reported in a series of CLL patients observed in the general practice and not enrolled in clinical trials [17]. The frequency of mutations involving the other investigated genes was in line with data published in literature using whole exome sequencing [41-44].

Interestingly, we observed that $18.0 \%$ of the cases presented more than one mutation. In the CLL11 trial, 161 patients were evaluated at the time of treatment requirement and NGS analysis revealed mutations in 42 out of 85 analyzed genes, with 76.4 and $42.2 \%$ of the patients presenting at least one or $\geq 2$ genes affected by mutations, respectively [14].

In our series of patients, the occurrence of mutations was associated with adverse molecular and genetic findings including $I G V H$ unmutated status, intermediate high-risk FISH results, and the presence of a complex karyotype. Noteworthy, a higher incidence of concurrent mutations was observed in TP53-mutated patients, while the presence of a complex karyotype was associated with TP53-, ATM-, and MYD88-mutated cases. These results suggest that concurrent mutations, as well as complex karyotype, might represent an aspect of genetic instability correlated to a defective DNA damage response [45].

We then analyzed the correlation between the mutational status and outcome. A shorter TTFT was observed in those patients with mutations by NGS and with mutations involving TP53, NOTCH1, ATM, and BRAF. The prognostic significance of BRAF mutations needs to be confirmed on larger series because it was derived from a limited number of patients, most of whom had concurrent mutations of other genes. By multivariate analysis, we found that the multi-hit profile ( $\geq 2$ mutations by NGS) was independently associated with a shorter TTFT along with TP53 disruption, IGHV unmutated status, and advanced stage. 


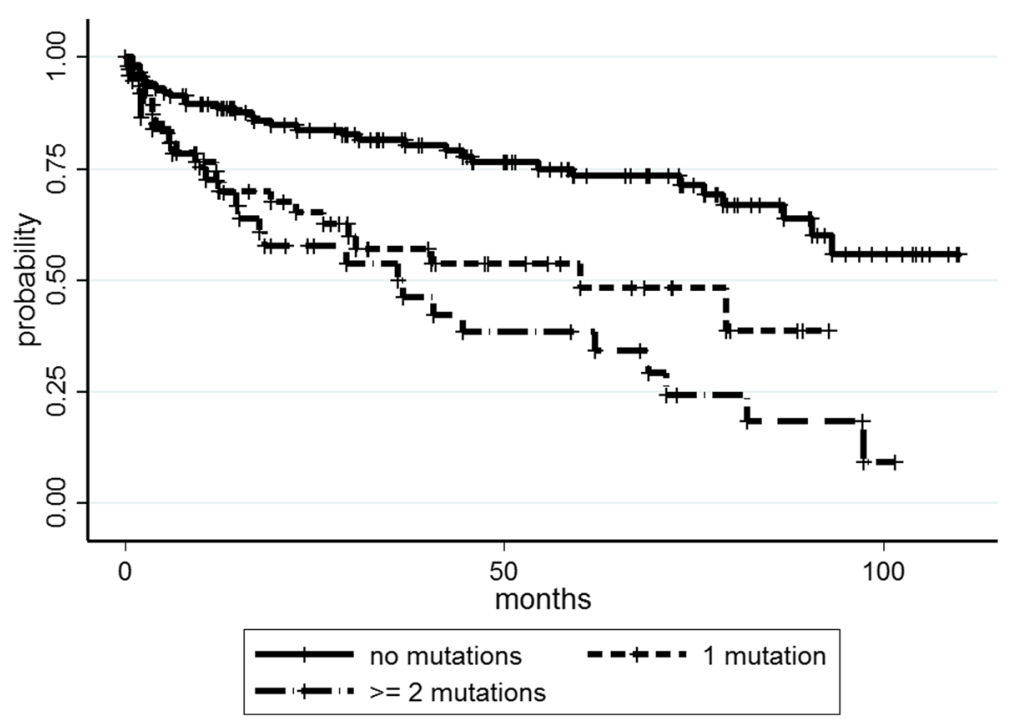

Fig. 2 TTFT according to number of mutations by NGS analysis $(p<0.001)$

Given the complexity of CLL genetic landscape, we suggest that not only the presence of clones or subclones [46] but also the concurrent presence of mutations may play a significant role in prognostication. This study, to our knowledge, provides the first demonstration that at diagnosis, in an unselected CLL patient population followed up at one center having a $>90 \%$ capture of incident cases, a multi-hit profile derived from an extensive NGS analysis is independently associated with a shorter TTFT. Noteworthy, concurrent gene mutations are also frequent in patients with relapsed/refractory CLL and are associated with a worse outcome [47].

When considering OS, a poorer outcome was associated with the presence of mutations by NGS, with mutations in TP53 and NOTCH1 genes, with the multi-hit profile, with
IGHV unmutated status, with TP53 disruption, and with the complex karyotype. However, by multivariate analysis, only TP53 disruption was independently associated with a worse outcome along with advanced stage, IGHV unmutated status, and the complex karyotype.

Whereas the strong independent impact on TTFT and OS of IGHV mutational status and TP53 disruption was previously demonstrated [12, 14, 15, 45, 46], the finding of an independent impact on OS of the complex karyotype is noteworthy, especially when considering that an extensive clinic-biologic characterization was performed in this patient cohort. Recently, an independent prognostic relevance on OS of the complex karyotype has emerged in CLL patients investigated at different phases of the disease: at diagnosis [13, 34], before first-line treatment [14],

Table 5 Multivariate analysis for TTFT and OS

\begin{tabular}{|c|c|c|c|c|c|c|c|c|c|c|}
\hline \multirow[b]{2}{*}{ Variable } & \multirow[b]{2}{*}{$H R$} & \multirow[b]{2}{*}{$\mathrm{Cl}$} & \multirow{2}{*}{$\begin{array}{l}\text { TTFT } \\
p\end{array}$} & \multicolumn{2}{|c|}{ After bootstrapping } & \multirow[b]{2}{*}{$H R$} & \multirow[b]{2}{*}{$\mathrm{Cl}$} & \multirow{2}{*}{$\begin{array}{l}\text { OS } \\
p\end{array}$} & \multicolumn{2}{|c|}{ After bootstrapping } \\
\hline & & & & $\mathrm{Cl}$ & $p$ & & & & $\mathrm{Cl}$ & $p$ \\
\hline Binet stage $b-c$ vs $a$ & 11.206 & $6.384-19.671$ & $<0.001$ & $5.570-22.545$ & $<0.001$ & 3.080 & $1.501-6.319$ & 0.002 & $1.302-7.286$ & 0.010 \\
\hline CD38 pos vs neg & 1.141 & $0.670-1.942$ & 0.627 & $0.663-1.938$ & 0.634 & 1.067 & $0.506-2.249$ & 0.864 & $0.448-2.356$ & 0.883 \\
\hline 11q deletion yes vs no & 1.306 & $0.619-2.755$ & 0.484 & $0.532-3.205$ & 0.560 & $\mathrm{Na}$ & $\mathrm{Na}$ & $\mathrm{Na}$ & $\mathrm{Na}$ & $\mathrm{Na}$ \\
\hline TP53 disruption yes vs no & 2.255 & $1.168-4.352$ & 0.015 & $1.039-4.891$ & 0.040 & 4.055 & $1.844-7.917$ & $<0.001$ & $1.897-8.670$ & $<0.001$ \\
\hline IGHV unmut vs mut & 5.078 & $2.599-9.554$ & $<0.001$ & $2.491-10.354$ & $<0.001$ & 3.198 & $1.524-6.13$ & 0.002 & $1.200-8.522$ & 0.020 \\
\hline \multicolumn{11}{|l|}{ No. of mutations by NGS } \\
\hline 0 & 1 & & & 1 & & 1 & & & 1 & \\
\hline 1 & 1.452 & $0.812-2.594$ & 0.208 & $0.574-3.673$ & 0.431 & 0.930 & $0.417-2.074$ & 0.860 & $0.348-2.484$ & 0.885 \\
\hline$\geq 2$ & 2.791 & $1.468-5.306$ & 0.002 & $1.375-5.665$ & 0.004 & 1.115 & $0.492-2.523$ & 0.795 & $0.480-2.589$ & 0.801 \\
\hline Complex karyotype yes vs no & 1.649 & $0.896-3.034$ & 0.108 & $0.824-3.301$ & 0.158 & 3.173 & $1.521-6.619$ & 0.002 & $1.369-7.355$ & 0.007 \\
\hline
\end{tabular}

f female, fav favorable, int intermediate, $m$ male, mut mutated, neg negative, pos positive, unfav unfavorable, unmut unmutated, yrs years, TP53 disruption $17 \mathrm{p} 13$ deletion and/or TP53 mutation 
and in refractory relapsed patients treated with ibrutinib [48]. We may assume that the complex karyotype probably reflects a high level of genomic instability that appears to be a better predictor of worse OS in comparison to single and multiple concurrent mutations, with the only exception of TP53 mutations. Thus, karyotyping seems to substantially contribute to the identification of CLL patients with most adverse prognosis and should be considered in an extensive diagnostic work-up in future CLL trials $[49,50]$.

\section{Conclusions}

Altogether, our data suggests that NGS may play an important role in the definition of the risk of disease progression and therefore could be useful in the diagnostic work-up of CLL patients as an efficient, sensitive, and affordable technique for routine screening of mutations. Indeed, NGS analysis, in combination with clinical stage, TP53 disruption, and IGHV assessment, may identify those patients that are at higher risk of progression and therefore need a stricter follow-up whereas karyotyping could represent along with TP53 disruption the best genetic predictor of OS. However, some issues need to be better defined before the introduction of the extensive NGS approach into the routine clinical practice: (i) which genes and how many genes should be included in the work-up panel for an efficient and affordable routine applicability, (ii) what cutoff for mutational analysis should be considered clinically relevant, and (iii) how to develop a standardized methodology ensuring reproducibility of the results [51].

\section{Additional files}

Additional file 1: Table S1. List of the mutations observed by NGS in the 84 mutated patients. (XLSX $22 \mathrm{~kb}$ )

Additional file 2: Table S2. Overview of the mutations observed in the 20 genes. (DOCX 13 kb)

Additional file 3: Table S3. Gene mutations according to the presence of 1 or $\geq 2$ mutations by NGS analysis. (DOC $33 \mathrm{~kb}$ )

\section{Abbreviations}

CLL: Chronic lymphocytic leukemia; FISH: Fluorescent in situ hybridization; IGHV: Immunoglobulin heavy chain gene; NGS: Next-generation sequencing; OS: Overall survival; PB: Peripheral blood; PGM: Personal Genome Machine; TTFT: Time to first treatment

\section{Acknowledgements}

Not applicable.

\section{Funding}

This study was supported by the FAR (Fondo di Ateneo per la Ricerca) 2012, 2013, 2014, and 2015 of the University of Ferrara (GMR, AC), Programma Ricerca Regione Università 2007-2009 University of Ferrara (GMR, AC), PRIN 2008 (AC), Ricerca Finalizzata (AC, project RF-2011-02349712), and AIL (Associazione Italiana contro le Leucemie Linfomi e Mieloma, Ferrara). EV and ES are supported by AlL-Ferrara.

\section{Availability of data and materials}

The datasets analyzed during the current study are available from the corresponding authors on reasonable request.

\section{Authors' contributions}

GMR, ES, MN, and AC conceived and designed the study. GMR, FMQ, MC, $S M, L F, E L, E V, E T, M A B, A M, A U$, and FC participated in the data acquisition and patients' follow-up. GMR, ES, CB, LL, MN, and AC carried out the analysis and interpretation of the data. All the authors contributed to the writing, approval, and review the manuscript.

\section{Competing interests}

The authors declare that they have no competing interests.

\section{Consent for publication}

Not applicable.

\section{Ethics approval and consent to participate}

The study was approved by the local ethics committee.

Comitato etico unico della provincia di Ferrara. Study no. 140399.

\section{Author details}

${ }^{1}$ Hematology Section, Department of Medical Sciences, Azienda Ospedaliero-Universitaria, Arcispedale S. Anna, University of Ferrara, Via Aldo Moro, 8, 44124 Ferrara, Cona, Italy. ${ }^{2}$ Department of Morphology, Surgery and Experimental Medicine, and "Laboratorio per le Tecnologie delle Terapie Avanzate" (LTTA), University of Ferrara, Ferrara, Italy.

Received: 19 August 2016 Accepted: 6 September 2016

Published online: 15 September 2016

\section{References}

1. Chiorazzi N, Rai KR, Ferrarini M. Chronic lymphocytic leukemia. N Engl J Med. 2005:352(8):804-15.

2. Zenz T, Mertens D, Küppers R, Döhner H, Stilgenbauer S. From pathogenesis to treatment of chronic lymphocytic leukaemia. Nat Rev Cancer. 2010;10(1):37-50.

3. Grever MR, Lucas DM, Dewald GW, Neuberg DS, Reed JC, Kitada S, et al. Comprehensive assessment of genetic and molecular features predicting outcome in patients with chronic lymphocytic leukemia: results from the US Intergroup Phase III Trial E2997. J Clin Oncol. 2007;25(7):799-804.

4. Hallek M. Chronic lymphocytic leukemia: 2015 update on diagnosis, risk stratification, and treatment. Am J Hematol. 2015;90(5):446-60.

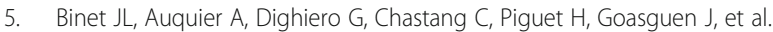
A new prognostic classification of chronic lymphocytic leukemia derived from a multivariate survival analysis. Cancer. 1981;48(1):198-206.

6. Damle RN, Wasil T, Fais F, Ghiotto F, Valetto A, Allen SL, et al. Ig V gene mutation status and CD38 expression as novel prognostic indicators in chronic lymphocytic leukemia. Blood. 1999;94(6):1840-7.

7. Wiestner A, Rosenwald A, Barry TS, Wright G, Davis RE, Henrickson SE, et al. ZAP-70 expression identifies a chronic lymphocytic leukemia subtype with unmutated immunoglobulin genes, inferior clinical outcome, and distinct gene expression profile. Blood. 2003;101(12):4944-51.

8. Bulian P, Shanafelt TD, Fegan C, Zucchetto A, Cro L, Nückel H, et al. CD49d is the strongest flow cytometry-based predictor of overall survival in chronic lymphocytic leukemia. J Clin Oncol. 2014;32(9):897-904.

9. Juliusson G, Oscier DG, Fitchett M, Ross FM, Stockdill G, Mackie MJ, et al. Prognostic subgroups in B-cell chronic lymphocytic leukemia defined by specific chromosomal abnormalities. N Engl J Med. 1990;323(11):720-4.

10. Döhner H, Stilgenbauer S, Benner A, Leupolt E, Kröber A, Bullinger L, et al. Genomic aberrations and survival in chronic lymphocytic leukemia. N Engl J Med. 2000;343(26):1910-6.

11. Cavazzini F, Hernandez JA, Gozzetti A, Russo Rossi A, De Angeli C, Tiseo R, et al. Chromosome 14q32 translocations involving the immunoglobulin heavy chain locus in chronic lymphocytic leukaemia identify a disease subset with poor prognosis. Br J Haematol. 2008;142(4):529-37.

12. Cuneo A, Rigolin GM, Bigoni R, De Angeli C, Veronese A, Cavazzini F, et al. Chronic lymphocytic leukemia with $6 \mathrm{q}$ - shows distinct hematological features and intermediate prognosis. Leukemia. 2004;18(3):476-83.

13. Rigolin GM, del Giudice I, Formigaro L, Saccenti E, Martinelli S, Cavallari M, et al. Chromosome aberrations detected by conventional karyotyping using 
novel mitogens in chronic lymphocytic leukemia: clinical and biologic correlations. Genes Chromosomes Cancer. 2015;54(12):818-26.

14. Herling CD, Klaumünzer M, Krings Rocha C, Altmüller J, Thiele H, Bahlo J, et al. Complex karyotypes, KRAS and POT1 mutations impact outcome in $\mathrm{CLL}$ after chlorambucil based chemo- or chemoimmunotherapy. Blood. 2016;128(3):395-404

15. Rossi D, Rasi S, Spina V, Bruscaggin A, Monti S, Ciardullo C, et al. Integrated mutational and cytogenetic analysis identifies new prognostic subgroups in chronic lymphocytic leukemia. Blood. 2013;121(8):1403-12.

16. Jeromin S, Weissmann S, Haferlach C, Dicker F, Bayer K, Grossmann V, et al. $S F 3 B 1$ mutations correlated to cytogenetics and mutations in NOTCH1, FBXW7, MYD88, XPO1 and TP53 in 1160 untreated CLL patients. Leukemia. 2014;28(1):108-17.

17. Baliakas P, Hadzidimitriou A, Sutton LA, Rossi D, Minga E, Villamor N, et al. Recurrent mutations refine prognosis in chronic lymphocytic leukemia. Leukemia. 2015;29(2):329-36.

18. Oscier DG, Rose-Zerilli MJ, Winkelmann N, Gonzalez de Castro D, Gomez B, Forster J, et al. The clinical significance of NOTCH1 and SF3B1 mutations in the UK LRF CLL4 trial. Blood. 2013;121(3):468-75.

19. Stilgenbauer S, Schnaiter A, Paschka P, Zenz T, Rossi M, Döhner K, et al. Gene mutations and treatment outcome in chronic lymphocytic leukemia: results from the CLL8 trial. Blood. 2014;123(21):3247-54

20. Pflug N, Bahlo J, Shanafelt TD, Eichhorst BF, Bergmann MA, Elter T, et al. Development of a comprehensive prognostic index for patients with chronic lymphocytic leukemia. Blood. 2014;124(1):49-62.

21. International CLL-IPI working group. An international prognostic index for patients with chronic lymphocytic leukaemia (CLL-IPI): a meta-analysis of individual patient data. Lancet Oncol. 2016;17(6):779-90.

22. Parikh SA, Strati P, Tsang M, West CP, Shanafelt TD. Should IGHV status and FISH testing be performed in all CLL patients at diagnosis? A systematic review and meta-analysis. Blood. 2016;127(14):1752-60.

23. Guièze R, Wu CJ. Genomic and epigenomic heterogeneity in chronic lymphocytic leukemia. Blood. 2015;126(4):445-53.

24. Rossi D, Bruscaggin A, Spina V, Rasi S, Khiabanian H, Messina M, et al. Mutations of the SF3B1 splicing factor in chronic lymphocytic leukemia: association with progression and fludarabine-refractoriness. Blood. 2011; 118(26):6904-8.

25. Landau DA, Tausch E, Taylor-Weiner AN, Stewart C, Reiter JG, Bahlo J, et al. Mutations driving CLL and their evolution in progression and relapse. Nature. 2015:526(7574):525-30.

26. Puente XS, Pinyol M, Quesada V, Conde L, Ordóñez GR, Villamor N, et al. Whole-genome sequencing identifies recurrent mutations in chronic lymphocytic leukaemia. Nature. 2011;475(7354):101-5.

27. Rossi $\mathrm{D}$, Khiabanian $\mathrm{H}$, Spina $\mathrm{V}$, Ciardullo $\mathrm{C}$, Bruscaggin $\mathrm{A}$, Famà $\mathrm{R}$, et al. Clinical impact of small TP53 mutated subclones in chronic lymphocytic leukemia. Blood. 2014;123(14):2139-47.

28. Malcikova J, Stano-Kozubik K, Tichy B, Kantorova B, Pavlova S, Tom N, et al. Detailed analysis of therapy-driven clonal evolution of TP53 mutations in chronic lymphocytic leukemia. Leukemia. 2015;29(4):877-85.

29. Rasi S, Khiabanian H, Ciardullo C, Terzi-di-Bergamo L, Monti S, Spina V, et al. Clinical impact of small subclones harboring NOTCH1, SF3B1 or BIRC3 mutations in chronic lymphocytic leukemia. Haematologica. 2016;101(4): e135-8.

30. Sutton LA, Ljungström V, Mansouri L, Young E, Cortese D, Navrkalova V, et al. Targeted next-generation sequencing in chronic lymphocytic leukemia: a high-throughput yet tailored approach will facilitate implementation in a clinical setting. Haematologica. 2015;100(3):370-6.

31. Vollbrecht C, Mairinger FD, Koitzsch U, Peifer M, Koenig K, Heukamp LC, et al. Comprehensive analysis of disease-related genes in chronic lymphocytic leukemia by multiplex PCR-based next generation sequencing. PLoS One. 2015;10(6):e0129544.

32. Hallek M, Cheson BD, Catovsky D, Caligaris-Cappio F, Dighiero G, Döhner H, et al. Guidelines for the diagnosis and treatment of chronic lymphocytic leukemia: a report from the International Workshop on Chronic Lymphocytic Leukemia updating the National Cancer Institute-Working Group 1996 guidelines. Blood. 2008;111(12):5446-56.

33. Matutes E, Owusu-Ankomah K, Morilla R, Garcia Marco J, Houlihan A, Que TH, et al. The immunological profile of B-cell disorders and proposal of a scoring system for the diagnosis of CLL. Leukemia. 1994;8(10):1640-5.

34. Rigolin GM, Cibien F, Martinelli S, Formigaro L, Rizzotto L, Tammiso E, et al. Chromosome aberrations detected by conventional karyotyping using novel mitogens in chronic lymphocytic leukemia with "normal" FISH: correlations with clinicobiologic parameters. Blood. 2012;119(10):2310-3.

35. Rigolin GM, Maffei R, Rizzotto L, Ciccone M, Sofritti O, Daghia G, et al. Circulating endothelial cells in patients with chronic lymphocytic leukemia: clinical-prognostic and biologic significance. Cancer. 2010;116(8):1926-37.

36. Bardi A, Cavazzini F, Rigolin GM, Tammiso E, Volta E, Pezzolo E, et al. Employment of oligodeoxynucleotide plus interleukin-2 improves cytogenetic analysis in splenic marginal zone lymphoma. J Biomed Biotechnol. 2011;2011:691493.

37. Rigolin GM, Saccenti E, Rizzotto L, Ferracin M, Martinelli S, Formigaro L, et al. Genetic subclonal complexity and miR125a-5p down-regulation identify a subset of patients with inferior outcome in low-risk CLL patients. Oncotarget. 2014;5(1):140-9.

38. Chang F, Li MM. Clinical application of amplicon-based next-generation sequencing in cancer. Cancer Genet. 2013:206(12):413-9.

39. Schnaiter A, Paschka P, Rossi M, Zenz T, Bühler A, Winkler D, et al. NOTCH1, SF3B1, and TP53 mutations in fludarabine-refractory CLL patients treated with alemtuzumab: results from the CLL2H trial of the GCLLSG. Blood. 2013; 122(7):1266-70.

40. Rossi D, Fangazio M, Rasi S, Vaisitti T, Monti S, Cresta S, et al. Disruption of BIRC3 associates with fludarabine chemorefractoriness in TP53 wild-type chronic lymphocytic leukemia. Blood. 2012;119(12):2854-62.

41. Wang L, Lawrence MS, Wan Y, Stojanov P, Sougnez C, Stevenson K, et al. SF3B1 and other novel cancer genes in chronic lymphocytic leukemia. N Engl J Med. 2011;365(26):2497-506.

42. Fabbri G, Rasi S, Rossi D, Trifonov V, Khiabanian H, Ma J, et al. Analysis of the chronic lymphocytic leukemia coding genome: role of NOTCH1 mutational activation. J Exp Med. 2011;208(7):1389-401.

43. Quesada V, Conde L, Villamor N, Ordóñez GR, Jares P, Bassaganyas L, et al. Exome sequencing identifies recurrent mutations of the splicing factor SF3B1 gene in chronic lymphocytic leukemia. Nat Genet. 2011;44(1):47-52.

44. Landau DA, Carter SL, Stojanov P, McKenna A, Stevenson K, Lawrence MS, et al. Evolution and impact of subclonal mutations in chronic lymphocytic leukemia. Cell. 2013;152(4):714-26.

45. Dicker F, Herholz H, Schnittger S, Nakao A, Patten N, Wu L, et al. The detection of TP53 mutations in chronic lymphocytic leukemia independently predicts rapid disease progression and is highly correlated with a complex aberrant karyotype. Leukemia. 2009;23(1):117-24.

46. Nadeu F, Delgado J, Royo C, Baumann T, Stankovic T, Pinyol M, et al. Clinical impact of clonal and subclonal TP53, SF3B1, BIRC3, NOTCH1, and ATM mutations in chronic lymphocytic leukemia. Blood. 2016;127(17):2122-30.

47. Guièze R, Robbe P, Clifford R, de Guibert S, Pereira B, Timbs A, et al. Presence of multiple recurrent mutations confers poor trial outcome of relapsed/refractory CLL. Blood. 2015;126(18):2110-7.

48. Thompson PA, O'Brien SM, Wierda WG, Ferrajoli A, Stingo F, Smith SC, et al. Complex karyotype is a stronger predictor than del(17p) for an inferior outcome in relapsed or refractory chronic lymphocytic leukemia patients treated with ibrutinib-based regimens. Cancer. 2015;121(20):3612-21.

49. Rossi D, Terzi-di-Bergamo L, De Paoli L, Cerri M, Ghilardi G, Chiarenza A, et al. Molecular prediction of durable remission after first-line fludarabinecyclophosphamide-rituximab in chronic lymphocytic leukemia. Blood. 2015; 126(16):1921-4

50. Cuneo A, Cavazzini F, Ciccone M, Daghia G, Sofritti O, Saccenti E, et al. Modern treatment in chronic lymphocytic leukemia: impact on survival and efficacy in high-risk subgroups. Cancer Med. 2014;3(3):555-64.

51. Pospisilova S, Gonzalez D, Malcikova J, Trbusek M, Rossi D, Kater AP, et al. ERIC recommendations on TP53 mutation analysis in chronic lymphocytic leukemia. Leukemia. 2012;26(7):1458-6. 